\title{
Complex Regional Pain Syndrome: Current Practice Management and Referral Trends in a Closed Healthcare System
}

\author{
Connor Zale ${ }^{1}$, Joshua Hansen ${ }^{2}$ (D) and Paul Ryan ${ }^{1, *(D)}$ \\ 1 Tripler Army Medical Center, Honolulu, HI 96859, USA; connor.l.zale.mil@mail.mil \\ 2 F. Edward Hebert School of Medicine, Uniformed Services University of Health Sciences, \\ Bethesda, MD 20814,USA; joshua.a.hansen34.mil@mail.mil \\ * Correspondence: paul.m.ryan.mil@mail.mil
}

Received: 4 November 2020; Accepted: 24 December 2020; Published: 31 December 2020

\begin{abstract}
Background: Complex regional pain syndrome (CRPS) is a neurologic condition that can present with severe pain and dysfunction. Delay in treatment adversely affects outcomes. The purpose of this study is to evaluate patient outcomes as they relate to the time from diagnosis to pain management referral once the diagnosis of CRPS has been made in a closed healthcare system. Methods: A retrospective record review from a closed healthcare system was utilized for CRPS cases from 2010-2019. Demographics, injury pattern, surgeries, pain score, treatment modalities, occupational outcomes, and time to pain management referral were recorded. Results: There were 26 cases of CRPS that met inclusion criteria. The mean time from diagnosis to treatment was 55 days. $16 / 26(61.5 \%)$ were medically discharged from the military. $23 / 26(88.5 \%)$ were unable to return to full duty due to CRPS. There was no significant difference in the reported pain scores over time regardless of treatment $(p=0.76)$. A linear regression demonstrated a significantly higher Visual Analog Scale Pain Score (VAS) over time in patients that were medically discharged $(p=0.022)$. Conclusions: The mean delay in referral to the pain service was 55 days. The majority of patients (88.5\%) did not return to full duty secondary to the diagnosis of CRPS, and $61.5 \%$ of patients required medical separation from active duty. Due to the negative impact that the diagnosis of CRPS has on occupational outcomes with a mean delay in referral of 55 days, clinics and providers should set up referral criteria and establish early pain pathways for patients diagnosed with CRPS.
\end{abstract}

Keywords: CRPS; pain management; complex regional pain syndrome

\section{Introduction}

Complex regional pain syndrome (CRPS) is a chronic neurologic condition of the extremities that can present with severe pain and nerve dysfunction including motor, sensory, and autonomic changes [1]. CRPS can be associated with trauma or elective surgery to the upper and lower limbs. In Type 1 CRPS, the injury or surgery does not directly damage the affected nerves. In Type 2 CRPS, there is a distinct injury to the nerve [2]. Despite the differing events that lead to CRPS, the symptoms are largely the same and can include swelling, stiffness, changes in coloration of the extremity, changes in temperature, and intense burning pain. Treatment for CRPS involves multimodal pain management utilizing corticosteroids, nonsteroidal anti-inflammatory medications (NSAIDs), and free radical scavengers (dimethylsulphoxide or $\mathrm{N}$-acetylcysteine) during the early stages of CRPS. Gabapentin, vasodilatory medications, physiotherapy, and sympathetic nerve blocks have also been used in the management of symptoms [3-5]. Narcotic pain medications have been found to be of little value in the care of CRPS [6]. The use of Vitamin C may also play a role in preventing the development of 
CRPS $[7,8]$. Early diagnosis and referral to physical therapy, occupational therapy, and chronic pain management is recommended to improve patient outcomes [9-11].

While early treatment and referral has been recommended, current practice management and referral trends are largely unknown. As it is a largely closed system, the Military Healthcare System provides an appropriate dataset from which management trends can be evaluated. The time from diagnosis to pain management referral within the military medical system is unknown. The rate of active-duty retention for military members with CRPS is currently unknown. The purpose of this study is to evaluate patient outcomes in relation to referral patterns in the treatment of CRPS.

\section{Materials and Methods}

This project was reviewed by the Tripler Army Medical Center IRB and approved as a quality improvement study. The military EMR (electronic medical record), AHLTA, was searched for all patients with ICD-9 codes 337.21 and 337.22 for reflex sympathetic dystrophy of the upper and lower limbs from 2010-2019 at a single institution. Minors and dependents were excluded from the study. Only active-duty service members with complete medical records were included in this study. Retirees that were diagnosed with CRPS after their service obligation were excluded, as there was the potential for medical visits outside of the military healthcare system for that population. Cases were reviewed using Budapest criteria [12,13]. Demographic information was collected to include the type of injury or surgery, date of injury or surgery, type and length of immobilization, visual analog pain score (VAS) at the time of diagnosis, referral to pain management, initial evaluation by pain management, and follow-up. The time from injury to CRPS diagnosis and the time from diagnosis to referral were obtained. The rate of return to full duty, the presence of formal duty or occupational limitations, and the rate of medical release from active duty were reviewed. CRPS treatment modalities were also recorded. Nonparametric and parametric statistical tests to include a Kruskal-Wallis rank sum test, ANOVA, student $t$-test, fisher exact test, and linear regression were performed using $\mathrm{R}$ statistical software version 3.6.0 (Free Software Foundation Inc. Boston, MA, USA). Linear modeling was performed for the VAS of patients with and without a medical board over time.

\section{Results}

43 cases were identified from the electronic medical record. Following a chart review, 17 cases were excluded. Of the 17 patients that were excluded, two patients were retirees, eight cases were dependents, one case developed CRPS prior to the EMR, and six cases were incorrectly coded. Following the exclusion, there 26 cases of CRPS were identified with active-duty military members. 23 cases $(88 \%)$ qualified for CRPS using the Budapest criteria. Two cases were diagnosed clinically by neurology, and one case was diagnosed by the primary care physician. The average age at the time of injury was 31.3 years old (20-49). There were 17 males (65\%) and 11 females (35\%). 18/26 (69\%) were immobilized for an average of 25 days (2-83). 12 cases involved an injury to an upper extremity (Table 1). 12 cases involved an injury to a lower extremity. One case occurred following an L5 corpectomy and posterior spinal fusion. One case resulted following a bilateral thoracoscopic sympathectomy for hyperhidrosis.

There was a mean of $55 \pm 109$ days from diagnosis of CRPS to referral to the pain management service (Table 2). The mean time from injury to diagnosis was $209 \pm 478$ months. The mean time from injury to referral was $209 \pm 479$ months. Table 3 demonstrates the occupational outcomes as they relate to the referral patterns. There was no significant difference in the time from CRPS diagnosis to pain management referral for cases that resulted in medical release from active duty (mean $=69 \pm 113$ days) when compared to those that were able to stay on active duty despite the diagnosis (mean $=32 \pm 94$ days) $(p=0.312)$. There was no significant difference from the time from injury to diagnosis for cases medically released from active duty (mean $=256 \pm 495$ months) when compared to cases where the patient was able to remain on active duty (mean $=135 \pm 504$ months) $(p=0.519)$. There was also no significant difference in the time from injury to referral to pain management for cases that resulted in a medical release from active duty (mean $=255 \pm 496$ 
months) when compared to cases where the patient was able to remain on active duty (mean $=135 \pm$ 505 months) $(p=0.521)$.

Table 1. Injury Patterns. The injury resulting in CRPS for each patient is displayed below.

\begin{tabular}{cc}
\hline Case & Injury \\
\hline 1 & Shoulder AC Joint Arthritis \\
2 & Partial Rotator Cuff Tear \\
3 & Thumb Ulnar Collateral Ligament Tear \\
4 & Forearm Crush and Compartment Syndrome \\
5 & Fifth Metacarpal Fracture \\
6 & Elbow Lateral Epicondylitis \\
7 & Distal Humerus Fracture \\
8 & Distal Radius Fracture \\
9 & Scaphoid Fracture and Perilunate Dislocation \\
10 & Cubital Tunnel Syndrome Surgery for Shrapnel to Shoulder \\
11 & Second Phalanx Fracture \\
12 & Bilateral Thoracoscopic Sympathectomy \\
13 & Bilateral Plantar Fasciitis \\
14 & Atraumatic Anterior Tibia Pain \\
15 & Patellofemoral Chondromalacia \\
16 & Stepped on a Sea Urchin \\
17 & Atraumatic Right Ankle Pain \\
18 & Lateral Ankle Sprain \\
19 & L5 Corpectomy and PSF L4-S1 \\
20 & Exertional Compartment Syndrome \\
21 & Cavus Foot Reconstruction \\
22 & Foot Pain after Ruck March \\
23 & Brachial Plexus Injury and T7 Compression Frability \\
24 & Base of the Fifth Metatarsal Avulsion \\
25 &
\end{tabular}

Table 2. Time to diagnosis and pain management referral. Time in days from diagnosis to pain management referral, time from injury to diagnosis, and time from injury to pain management referral are displayed below.

\begin{tabular}{cccc}
\hline Patient & $\begin{array}{c}\text { Time from Diagnosis } \\
\text { to Referral }\end{array}$ & $\begin{array}{c}\text { Time from Injury } \\
\text { to Diagnosis }\end{array}$ & $\begin{array}{c}\text { Time from Injury } \\
\text { to Referral }\end{array}$ \\
\hline 1 & 0 & 686 & 686 \\
2 & 98 & 148 & 50 \\
3 & 355 & 419 & 64 \\
4 & 6 & 60 & 54 \\
5 & 0 & 613 & 613 \\
6 & 0 & 1888 & 1888 \\
7 & 14 & 144 & 158 \\
8 & 0 & 130 & 130 \\
9 & 0 & 60 & 60 \\
10 & 0 & 375 & 375 \\
11 & 6 & 61 & 67 \\
12 & 105 & 19 & 124 \\
13 & 0 & 57 & 57 \\
14 & 32 & 38,649 & 38,617 \\
15 & 50 & 40,552 & 40,502 \\
16 & 0 & 39,011 & 39,011 \\
17 & 23 & 78 & 55 \\
18 & 442 & 38,712 & 39,154 \\
19 & 0 & 580 & 580 \\
20 & 109 & 159 & 50 \\
21 & 0 & 56 & 56 \\
22 & 15 & 45 & 30 \\
23 & 0 & 97 & 97 \\
24 & 6 & 73 & 67 \\
25 & 54 & 180 & 126 \\
26 & 104 & 359 & 255 \\
\hline
\end{tabular}


Table 3. Compares the occupational outcomes in terms of time differences from injury to diagnosis to referral.

\begin{tabular}{cccc}
\hline & $\begin{array}{c}\text { Remained on Active Duty } \\
\text { (Mean } \pm \text { std Deviation) }\end{array}$ & $\begin{array}{c}\text { Medical Release } \\
\text { (Mean } \pm \text { std Deviation) }\end{array}$ & $p$ Value \\
\hline Diagnosis to referral (days) & $32 \pm 94$ & $69 \pm 113$ & 0.312 \\
Injury to diagnosis (days) & $135 \pm 504$ & $256 \pm 495$ & 0.519 \\
Injury to referral (days) & $135 \pm 505$ & $255 \pm 496$ & 0.521 \\
\hline
\end{tabular}

Treatment pathways were patient- and provider-specific. All 26 patients went to physical therapy or occupational therapy for desensitization therapy. Eight of 26 patients underwent acupuncture. Two of 26 patients were referred to a chiropractor. Twenty-one of 26 took gabapentin or pregabalin. Twelve were treated with chronic narcotics. Eleven were taking acetaminophen for pain. Eleven were prescribed an NSAID. Three were prescribed duloxetine, and nine were prescribed amitriptyline. Only one patient was prescribed vitamin C. Eleven used topical lidocaine. Ten received a stellate ganglion or lumbar sympathetic nerve block. Four had a peripheral nerve block. Four underwent a spinal cord stimulator. Chronic opioid use was statistically higher among patients that underwent a medical board $(p=0.042)$ (Table 4$)$. There was no significant difference in the reported pain scores over time regardless of the time to referral, as demonstrated in Figure $1(p=0.76)$.

Table 4. The treatment type and $p$ value for patients that remained on active duty and underwent a medical board are displayed below.

\begin{tabular}{ccccc}
\hline & All $\mathbf{( N = 2 6 )}$ & $\begin{array}{c}\text { Remained on Active } \\
\text { Duty } \mathbf{( N = 1 0 )}\end{array}$ & $\begin{array}{c}\text { Medical Release } \\
\mathbf{( N = 1 6 )}\end{array}$ & $p$ Value \\
\hline Physical therapy & $26 / 26$ & 10 & 16 & 1 \\
Gabapentin & $21 / 26$ & 8 & 13 & 0.657 \\
Chronic narcotics & $12 / 26$ & 2 & 10 & 0.042 \\
Acetaminophen & $11 / 26$ & 4 & 7 & 0.589 \\
NSAIDs & $11 / 26$ & 4 & 7 & 0.589 \\
Topical lidocaine & $11 / 26$ & 4 & 7 & 0.589 \\
Amitripyline & $10 / 26$ & 4 & 6 & 0.609 \\
Acupuncture & $9 / 26$ & 3 & 6 & 0.517 \\
Peripheral nerve block & $8 / 26$ & 1 & 3 & 0.081 \\
Spinal cord stimulator & $4 / 26$ & 1 & 3 & 0.496 \\
Duloxetine & $4 / 26$ & 1 & 3 & 0.496 \\
Vitamin C & $3 / 26$ & 0 & 0 & 0.215 \\
\end{tabular}

Twenty-three of 26 patients (88.5\%) were given occupational restrictions secondary to the diagnosis. Sixteen $(61.5 \%)$ were medically released from active duty secondary to the diagnosis of CRPS. A linear modeling with ANOVA was performed for the VAS over time for those patients who required a medical release from active duty. The linear modeling with ANOVA demonstrated that there was a significantly higher VAS over time for patients who required a medical discharge from active duty when compared to those who were able to remain on active duty, as shown in Figure $2(p=0.022)$. The mean VAS, however, was not significantly different between those patients who were medically released from active duty and those who were not $(p=0.207)$. 


\section{VAS for CRPS Patients}

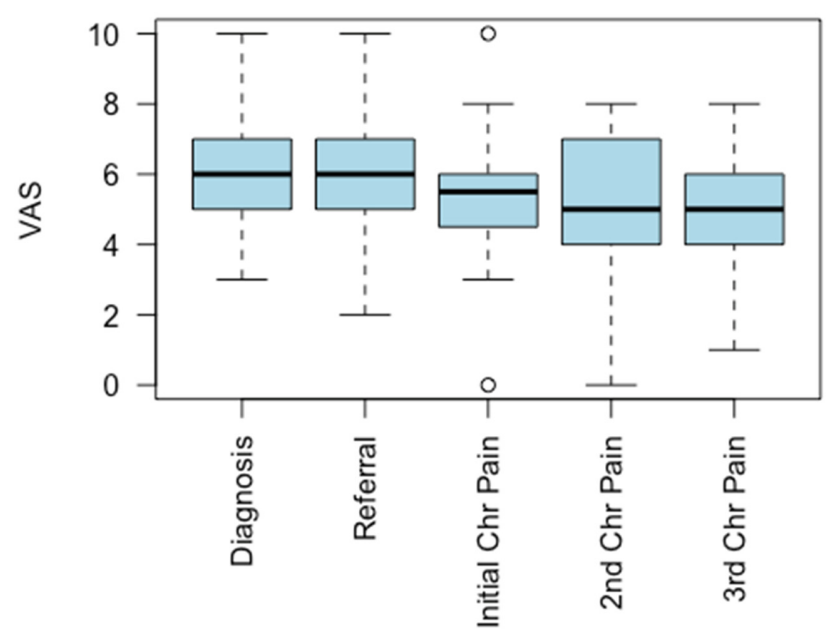

Figure 1. Pain scores over time. Displayed above is the VAS for all CRPS patients at each appointment over time. VAS at time of diagnosis (Diagnosis), initial referral to chronic pain management (Referral), initial evaluation at chronic pain management (Initial Chr Pain), follow-up appointment with chronic pain management (2nd Chr Pain), and second follow-up appointment with chronic pain management (3rd Chr Pain) are displayed above. Dots demonstrate outliers greater than or less than 1.5 times the upper and lower quartiles.

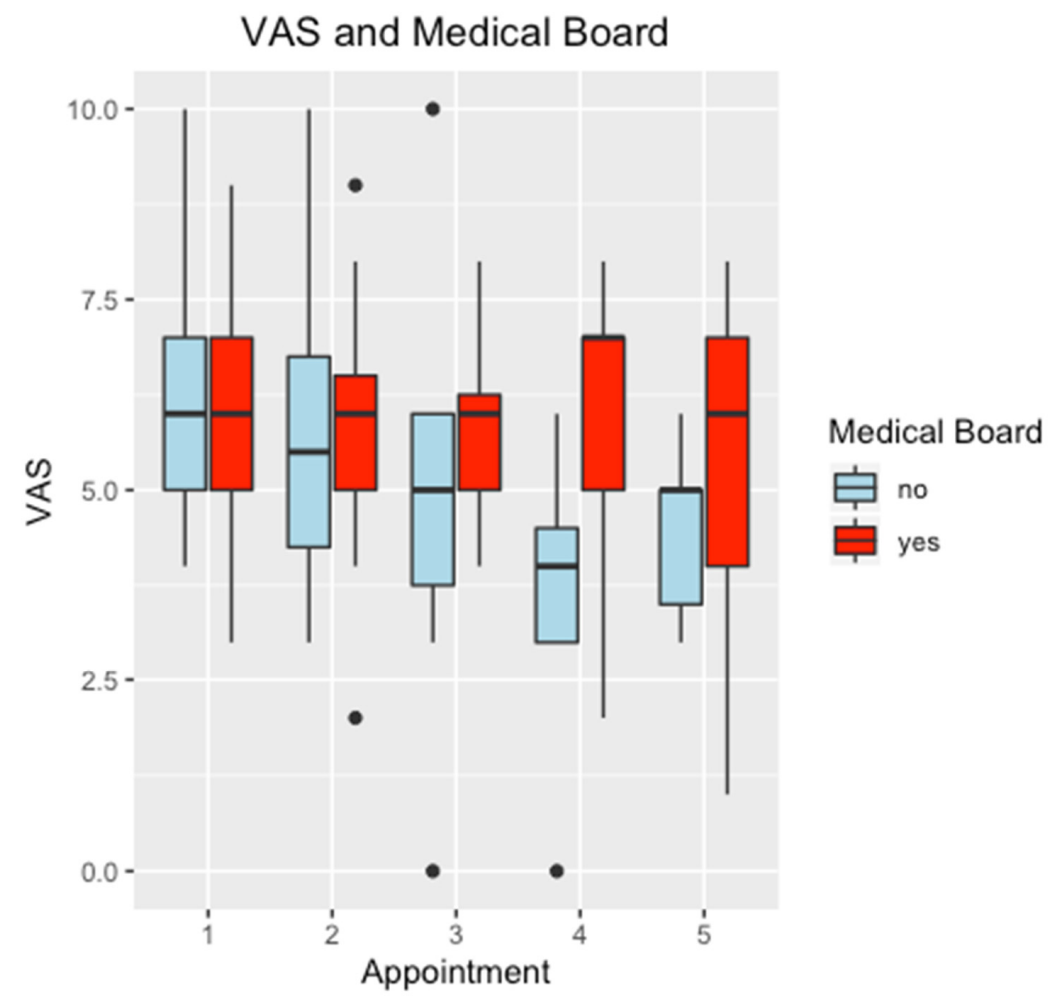

Figure 2. VAS and medical board results. Displayed above is the VAS according to the medical board status for patients over time. The VAS at time of diagnosis (Appointment 1), initial referral to chronic pain management (Appointment 2), initial evaluation at chronic pain management (Appointment 3), follow-up appointment with chronic pain management (Appointment 4), and second follow-up appointment with chronic pain management (Appointment 5) are displayed above. Dots demonstrate outliers greater than or less than 1.5 times the upper and lower quartiles. 


\section{Discussion}

Complex regional pain syndrome is a clinical disorder that results from peripheral and central responses to an injury. The clinical presentation can be variable, with hypersensitivity to painful and nonpainful stimuli. Sweating may be asymmetric to the contralateral limb. There may be color and skin changes. Hair growth and nail growth may be abnormal. The temperature of the skin may be warmer or colder than the contralateral limb on examination [5,11,12]. The International Association for the Study of Pain (IASP) developed diagnostic criteria for CRPS in 1993. A diagnosis of CRPS required the following: (1) a noxious event or immobilization to start the process, (2) allodynia, hyperalgesia, or pain out of proportion compared to the precipitating event, (3) the presence of edema, changes in skin blood flow, or abnormal sudomotor activity, and (4) the diagnosis can be excluded if the presence of this kind of pain and dysfunction could be related to another cause. In 2003, the IASP revised the diagnostic criteria of CRPS in Budapest. The new diagnostic criteria included patient-reported symptoms as well as a clinical evaluation of sensory, vasomotor, sudomotor/edema, and motor/trophic findings [12,13]. Despite standardized criteria according to the IASP or the Budapest Criteria, there is variable diagnostic consistency among clinicians for the diagnosis of CRPS (IASP kappa $=0.43-0.66$ and Budapest kappa $=0.66-0.69)$ [13]. Similar to other studies, the patients in our study were not selected based on strict IASP standards. We included any patient who was diagnosed and treated for CRPS due to the range of clinical symptoms and various diagnostic systems developed. In some cases, the patients were diagnosed prior to referral, and in other cases the diagnosis was made after referral to the pain service. The charts for all 26 patients were reviewed as part of this study, and 23 of the 26 patients did meet the Budapest Criteria. The remaining three cases were diagnosed clinically. Two cases were diagnosed by a neurologist, and one case was diagnosed by the primary physician. Each of the three cases that did not meet the Budapest Criteria lacked only one documented clinical symptom. As this was a retrospective chart review, it is possible that the symptom existed but was not queried or was not documented.

Early diagnosis and treatment are recommended to avoid disuse and worsening of the patients' psychological and physical condition. Early referral to therapy is recommended for gentle movement as well as an interdisciplinary approach with "four pillars of intervention" [5]. The pillars of intervention include patient education, pain relief with medication and procedures, physical rehabilitation, and psychological intervention. Treatment should be tailored to the most prominent symptoms of each case of CRPS [11]. If there is prominent inflammation, then NSAIDs should be used. Analgesics are prescribed for pain and sensory disorders. Vasodilators, such as calcium channel blockers, alpha sympathetic blockers, or phosphodiesterase inhibitors, can be used for vasomotor disturbances, and muscle relaxants can be prescribed in cases of motor disorders. Psychological interventions are useful for cases with psychological problems such as chronic pain behavior, depression, or anxiety. In our limited study population with large variabilities in the referral patterns, we did not find a significant difference in the occupational outcomes or visual analog pain scales in patients based upon the time to referral to a pain management service.

A systematic review was performed in 2010 to develop multidisciplinary guidelines for the treatment of CRPS-1 [14]. It found no evidence for the use of paracetamol (acetaminophen) in treating CRPS-1 pain (level 4). Insufficient evidence was found to support the use of NSAIDs to control pain (level 3). There was insufficient evidence to determine the effects of oral opioids on pain in patients with CRPS (level 3). Gabapentin administered at 600-1800-mg doses every $24 \mathrm{~h}$ was found to reduce pain symptoms in the first eight weeks (level 2). Dimethylsulphoxide cream (DMSO) was found to reduce symptoms in CRPS-1 patients (level 2). $\mathrm{N}$-acetylcysteine at $600 \mathrm{mg}$ three times a day also reduced symptoms (level 3). In addition, there was no evidence that antidepressants were effective in reducing pain in CRPS-1 patients (level 4). Physiotherapy was found to have a positive effect for patients with CRPS (level 2). The majority of patients in our study population were treated with gabapentin (21/26), and all patients (26/26) were referred to physiotherapy. 
A recent review found that published studies on the pharmacologic treatment of CRPS are primarily underpowered and heterogenous in terms of patient demographics and drug doses [15]. Specific conclusions for the pharmacologic management of CRPS could not be made. Patient demographics, the variable range of symptoms and clinical findings, as well as the lack of clinical evidence, makes it difficult to develop standardized treatment protocols for CRPS. For patients that fail pharmacologic, physical, and psychological therapy, invasive treatments may be pursued. Invasive procedures include sympathetic blocks (stellate ganglion and lumbar sympathetic blocks), spinal blocks, peripheral nerve blocks, radiofrequency ablation neurolysis, phenol neurolysis, or spinal cord stimulators $[11,16]$.

In our patient population, various treatment modalities were employed for all 26 patients using an interdisciplinary team. This study consisted of a younger patient population with heterogenous injuries. None of the patients in our cohort were prescribed Vitamin C prophylactically for CRPS. One case was prescribed Vitamin C after the diagnosis of CRPS was made. Vitamin C can be prescribed at $500 \mathrm{mg}$ for 45-50 days immediately after the time of injury or surgery in cases in which the orthopedic surgeon is suspicious of a possible development of CRPS $[7,8,11]$. While a prompt referral to a pain specialist is recommended, some treatments can be initiated by the referring provider if there is a delay in initiating a pain service consultation. Recommendations exist for the initiation of gabapentin, NSAIDs, and acetaminophen as initial treatments.

This is the first study to evaluate injuries associated with CRPS and the time to pain management referral in a closed healthcare system. There is a paucity of literature with regards to CRPS in the military, with a single case report and a case series documenting CRPS in lumbosacral plexopathy $[17,18]$. Our study demonstrated a $62 \%$ medical separation rate for active-duty personnel who developed CRPS. Linear regression modeling determined a significant decrease in the VAS over time for patients who were able to remain on active duty when compared to patients who were medically discharged. With the available numbers, we were unable to determine if medical discharge occurred for cases that did not demonstrate a positive response to pain management or if these cases had a greater severity of CRPS at baseline.

The limitations of this study include its retrospective nature, small sample size, and heterogeneity of treatments. We did not evaluate the severity of CRPS reported by the patient and provider on their return to activity or work prior to and following interdisciplinary pain management. Due to the small sample size, we were unable to evaluate the rate of medical discharge for military personnel as it related to their individual work requirements as defined by their military occupation specialty code (MOS). The variability in the time from diagnosis to referral and in the time from diagnosis to treatment reflects the lack of a CRPS clinical pathway in the studied healthcare system. Since treatments were patient- and provider-specific, the variations in the treatment processes that occurred were larger than expected. The large variances in this study limit the ability to draw outcome-specific solutions with the current sample size. With that said, the results demonstrate that a clinical practice guideline or published guideline may help improve patient care, especially in the treatment of conditions that are seen less commonly by referring providers.

An early diagnosis and referral to chronic pain management has been recommended to improve patient-reported pain and functional outcomes through the mitigation of disuse and worsening psychological and physical impairment. The development of CRPS can have an effect on occupational outcomes, as evidenced by the return to duty rates for affected service members. Although we were not able to demonstrate a difference in occupational outcomes in relation to the time to referral, $62 \%$ of active-duty service members with complex regional pain syndrome were medically discharged regardless of the time to referral. In this study, there was a mean delay in referral to a specialty pain service of 55 days. Due to the impact that CRPS has on occupational outcomes as measured by retention on active duty, clinics and providers should set up referral criteria and establish early pain pathways for patients who have been diagnosed with CRPS. Larger studies examining the effect of clinical practice guidelines may help to establish improved patient outcomes in the treatment of CRPS. 
Author Contributions: Conceptualization, P.R. and C.Z.; methodology, C.Z.; software, C.Z.; validation, P.R., C.Z. and J.H.; formal analysis, P.R., C.Z., and J.H.; investigation, C.Z. and J.H.; resources, C.Z. and P.R.; data curation, C.Z. and J.H.; writing-original draft preparation, C.Z. and J.H.; writing-review and editing, P.R.; visualization, P.R.; supervision, P.R.; project administration, P.R.; All authors have read and agreed to the published version of the manuscript.

Funding: This research received no external funding.

Institutional Review Board Statement: This project was reviewed by the Tripler Army Medical Center Institutional Review Board and approved as a Quality Improvement Project.

Informed Consent Statement: Patient consent was not required due to the restrospective nature and limited scope of the chart review.

Data Availability Statement: The data presented in this study are available on request from the corresponding author. The data are not publicly available due to the study performed with military individual electronic medical records.

Conflicts of Interest: The authors declare no conflict of interest. The views expressed in this abstract/manuscript are those of the author(s) and do not reflect the official policy or position of the Uniformed Services University of the Health Sciences, Department of the Army, Department of Defense, or the US Government.

\section{References}

1. Goh, E.L.; Chidambaram, S.; Ma, D. Complex regional pain syndrome: A recent update. Burn. Trauma 2017, 5. [CrossRef] [PubMed]

2. Guthmiller, K.B.; Varacallo, M. Complex Regional Pain Syndrome (CRPS), Reflex Sympathetic Dystrophy (RSD). In StatPearls; StatPearls Publishing: Treasure Island, FL, USA, 2020.

3. Birklein, F.; Dimova, V. Complex regional pain syndrome-Up-to-date. Pain Rep. 2017, 2. [CrossRef] [PubMed]

4. Norli, E.S.; Brinkmann, G.H.; Kvien, T.K.; Bjørneboe, O.; Haugen, A.J.; Nygaard, H.; Mjaavatten, M.D. Standards for the diagnosis and management of complex regional pain syndrome: Results of a European Pain Federation task force. Eur. J. Pain 2019, 23, 641-651. [CrossRef]

5. Turner-Stokes, L.; Goebel, A. Complex regional pain syndrome in adults: Concise guidance. Clin. Med. 2011, 11, 596-600. [CrossRef] [PubMed]

6. Hogan, C.J.; Hurwitz, S.R. Treatment of complex regional pain syndrome of the lower extremity. J. Am. Acad. Orthop. Surg. 2002, 10, 281-289. [CrossRef] [PubMed]

7. Aim, F.; Klouche, S.; Bauer, T.; Hardy, P. Efficacy of vitamin C in preventing complex regional pain syndrome after wrist fracture: A systematic review and meta-analysis. Orthop. Traumatol. Surg. Res. 2017, 103, 465-470. [CrossRef] [PubMed]

8. Shibuya, N.; Humphers, J.; Agarwal, M.; Jupiter, D. Efficacy and Safety of High-dose Vitamin C on Complex Regional Pain Syndrome in Extremity Trauma and Surgery Systematic Review and Meta-Analysis. J. Foot Ankle Surg. 2013, 52, 62-66. [CrossRef] [PubMed]

9. De Mos, M.; Huygen, F.J.P.M.; Van Der Hoeven-Borgman, M.; Dieleman, J.P.; Stricker, B.H.C.; Sturkenboom, M.C.J.M. Referral and treatment patterns for complex regional pain syndrome in the Netherlands. Acta Anaesthesiol. Scand. 2009, 53, 816-825. [CrossRef] [PubMed]

10. Grieve, S.; Llewellyn, A.; Jones, L.; Manns, S.; Glanville, V.; McCabe, C.S. Complex regional pain syndrome: An international survey of clinical practice. Eur J. Pain 2019, 23, 1890-1903. [CrossRef] [PubMed]

11. Bharwani, K.D.; Dirckx, M.; Huygen, F.J. Complex regional pain syndrome: Diagnosis and treatment. BJA Educ. 2017, 17, 262-268. [CrossRef]

12. Iolascon, G.; de Sire, A.; Moretti, A.; Gimigliano, F. Complex regional pain syndrome (CRPS) type I: Historical perspective and critical issues. Clin. Cases Miner. Bone Metab. 2015, 12 (Suppl. 1), 4-10. [CrossRef]

13. Harden, R.N.; Bruehl, S.; Perez, R.S.; Birklein, F.; Marinus, J.; Maihofner, C.; Mogilevski, M. Validation of proposed diagnostic criteria (the "Budapest Criteria") for Complex Regional Pain Syndrome. Pain 2010, 150, 268-274. [CrossRef] [PubMed]

14. Perez, R.S.; Zollinger, P.E.; Dijkstra, P.U.; Thomassen-Hilgersom, I.L.; Zuurmond, W.W.; Rosenbrand, K.C.; Geertzen, J.H. Evidence based guidelines for complex regional pain syndrome Type 1. BMC Neurol. 2010, 10,1-14. [CrossRef] [PubMed]

15. Misidou, C.; Papagoras, C. Complex Regional Pain Syndrome: An Update. Mediterr. J. Rheumatol. 2019, 30, 16-25. [CrossRef] [PubMed] 
16. Lo, J.; Cavazos, J.; Christopher, B. Management of Complex Regional Pain Syndrome. In Baylor University Medical Center Proceedings; Taylor \& Francis: Abingdon, UK, 2017; Volume 30, pp. 286-288.

17. Gallo, A.C.; Codispoti, V.T. Complex regional pain syndrome type II associated with lumbosacral plexopathy: A case report. Pain Med. 2010, 11, 1834-1836. [CrossRef] [PubMed]

18. Verdolin, M.H.; Stedje-Larsen, E.T.; Hickey, A.H. Ten consecutive cases of complex regional pain syndrome of less than 12 months duration in active duty United States military personnel treated with spinal cord stimulation. Anesth. Analg. 2007, 104, 1557-1560. [CrossRef] [PubMed]

Publisher's Note: MDPI stays neutral with regard to jurisdictional claims in published maps and institutional affiliations.

(C) 2020 by the authors. Licensee MDPI, Basel, Switzerland. This article is an open access article distributed under the terms and conditions of the Creative Commons Attribution (CC BY) license (http://creativecommons.org/licenses/by/4.0/). 